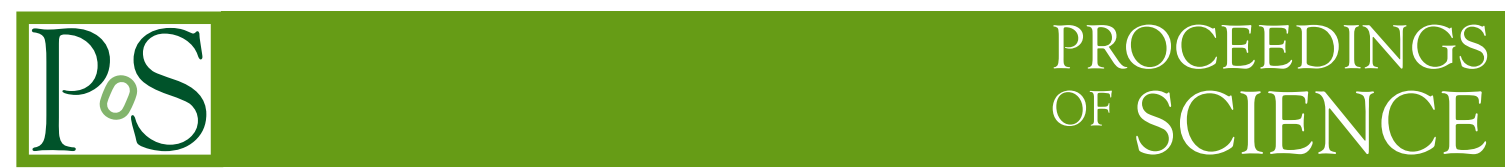

\title{
Scale symmetry without the anomaly
}

\author{
Paweł Olszewski* \\ Institute of Theoretical Physics, Faculty of Physics, University of Warsaw, Poland \\ E-mail: Pawel.Olszewski@fuw.edu.pl
}

\begin{abstract}
Nonrenormalizable lagrangians can describe models with spontaneously broken and nonanomalous scale symmetry. This characteristic is a regularization condition and can be conveniently imposed with the help of scale invariant regularization method. We present this method at an example of a simple toy-model and discuss how usage of the method results in modifications of renormalization group functions relative to those obtained in regular dimensional regularization.
\end{abstract}

Corfu Summer Institute 2016 "School and Workshops on Elementary Particle Physics and Gravity" 31 August - 23 September, 2016

Corfu, Greece

${ }^{*}$ Speaker. 


\section{Introduction}

We discuss a way of regularizing loop corrections to effective action that does not spoil the scale symmetry of classical action. The method is based on using dimensional regularization but with a dynamical scalar field in the role of renormalization scale $\mu$. In the next section we recall basic definitions of the effective action and scale symmetry. In the third section we specify the discussion to the case of a simple Lagrangian with two interacting scalar fields and use it to explicitly introduce the scale invariant method of regularization. The discussed model is nonrenormalizable and it is shown to describe interactions after a spontaneous breakdown of the scale symmetry. The fourth section deals with renormalization of the model. We introduce counterterms and discuss renormalization group functions in this scale invariant setup.

\section{Quantum corrections and scale symmetry}

Consider calculating effective action $\Gamma[\Phi]$, the generating functional of 1-PI correlation functions, in a perturbative expansion both in powers of coupling constants $\lambda$ (or in the number of loops) as well as in powers of momentum of the $\Phi$ field configuration,

$$
\begin{aligned}
S[\Phi] & =\int \mathrm{d}^{4} x \mathscr{L}_{\text {class }}(\Phi), \quad \mathrm{e}^{i W[J ; \varphi]} \equiv \int \mathscr{D} \Phi \mathrm{e}^{i(S[\Phi+\varphi]+\Phi J)} \\
\Gamma[\Phi ; \varphi] & \equiv W\left[J_{\Phi} ; \varphi\right]-J_{\Phi} \Phi,\left.\quad \frac{\delta W}{\delta J}\right|_{J=J_{\Phi}}=\Phi, \quad \Gamma[\Phi ; 0]=\int \mathrm{d}^{4} x \mathscr{L}_{\text {eff }}(\Phi) \\
\mathscr{L}_{\text {eff }}(\Phi) & =-V_{\text {eff }}(\Phi)+\mathscr{Z}(\Phi)(\partial \Phi)^{2}+(\text { higher derivatives })
\end{aligned}
$$

To the lowest order, effective Lagrangian is equal to the classical one, $\mathscr{L}_{\text {eff }}=\mathscr{L}_{\text {class }}+\mathscr{O}(\hbar)$. An useful way of computing corrections to $\mathscr{L}_{\text {eff }}$ is the background field method. It relies on the fact that $\Gamma[\Phi+\varphi ; 0]=\Gamma[\Phi ; \varphi]$. The $\varphi$ function is called the background field. To compute $\Gamma[\Phi ; 0]$ one splits the $\Phi(x)$ variable into momentumless $\Phi_{0}$ and the fluctuation $\Phi^{\prime}$, where the latter is defined to vanish if not hit by a space-time derivative,

$$
\Phi=\Phi_{0}+\Phi^{\prime}, \quad \Gamma[\Phi ; 0]=\Gamma\left[\Phi^{\prime} ; \Phi_{0}\right]
$$

It follows that the corrections can be calculated using $\Phi_{0}$-dependent Feynman rules in momentum space and treating $\Phi^{\prime}$ as the field on internal and external lines. The sum of diagrams is then expanded in powers of the external momenta and consecutive terms of that expansion determine the functions in (2.3). $V_{e f f}$ is given by the sum of vacuum diagrams. See [1,2] for a detailed presentation of 1-PI effective action in general and $[4,5]$ for discussion of the momentum expansion.

It may happen that a transformation of fields that is a symmetry of $S[\Phi]$ is not a symmetry of $\Gamma[\Phi]$. The symmetry is then called anomalous.

Consider the scale transformation (dilatation) of a scalar field in $D=4-2 \varepsilon$ space-time dimensions,

$$
\begin{aligned}
\Phi(x) & \rightarrow s^{d_{\Phi}} \Phi(s x), \quad \delta \Phi(x)=\left(d_{\Phi}+x^{v} \partial_{v}\right) \Phi(x), \quad d_{\Phi}=1-\varepsilon \\
\mathscr{L}_{\text {class }} & =\frac{1}{2}(\partial \Phi)^{2}-V^{(0)}(\Phi) .
\end{aligned}
$$


It generalizes to fields with higher spins in an obvious way.

An action (classical or effective) is invariant with respect to dilatations when

$$
\delta \mathscr{L}(\Phi(x))=\left(D+x^{v} \partial_{v}\right) \mathscr{L}(\Phi(x))=\partial_{v}\left(x^{v} \mathscr{L}(\Phi(x))\right)
$$

For $\mathscr{L}_{\text {class }}$ in $D=4$ dimensions this simply requires that $V^{(0)}$ does not contain any dimensionful parameters. But, assuming a quantum field theory under consideration is not free of UV divergencies, one needs to rely on a regularization procedure to compute $\Gamma[\Phi]$. This generically necessitates introducing a dimensionful parameter $M$ that functions as a regulator of divergent loop integrals. $M$ eventually appears in $\mathscr{L}_{\text {eff }}(\phi)$, for example as an argument of the logarithm function

$$
\mathscr{L}_{\text {eff }} \supset \lambda^{2} \Phi^{4} \log \frac{\Phi}{M}
$$

at the one-loop level. The regulator, by assumption, is not charged under dilatations,

$$
\delta M=0
$$

Hence $\mathscr{L}_{\text {eff }}$ does not satisfy (2.7) and in effect the scale symmetry is anomalous. If one had assumed that the scale symmetry of $\mathscr{L}_{\text {class }}$ was spontaneously broken, then - at the lowest order in his perturbative calculation - he would observe presence of a massless mode, the Goldstone boson. However including loop corrections would reveal that the would-be-Goldstone mode is massive. Thus, absence of the scale symmetry has a physical consequence.

\section{Scale invariant regularisation}

The idea that prompts one to consider constructing truly scale symmetric models is a scale invariant (SI) regularization. The proposition is to use a regulator that is a dynamical quantum field charged under dilatations rather than an inert parameter. It is an established fact that, when using ordinary regularization methods (like momentum cut-off or dimensional regularization), physical predictions are the same regardless of which method was used. A similar conjecture must be true for regularization methods with a dynamical regulator. No such conjecture was proven and there is not a variety of dynamical regulators discussed in literature so far. A lattice regularization, where the fluctuation of the lattice spacing is a propagating and interacting degree of freedom, was proposed in [3]. But the only commonly discussed SI regularization is one inspired by dimensional regularization where the renormalization scale $\mu$ is promoted to be a function of quantum fields, $\mu=\mu(\Phi)$. I was originally proposed in [6, 7] and more recently used also in $[8,9,10]$.

We will discuss the latter SI regularization method using the example of a toy-model with two interacting scalar fields. Related considerations that employ a similar model but not a SI regularization can be found in [11]. The connection between scale symmetry and nonrenormalizable interactions was previously discussed in $[12,13]$. An extension of the Standard Model with the dimensional SI regularization was considered in [14]. 
Consider the following classical Lagrangian in $D=4-2 \varepsilon$ dimensions for fields $\phi$ and $\sigma$.

$$
\begin{aligned}
& \mathscr{L}_{\text {class }}=\frac{1}{2}(\partial \phi)^{2}+\frac{1}{2}(\partial \sigma)^{2}-\left(z M_{2}^{\frac{1}{1-\varepsilon}}\right)^{2 \varepsilon} F\left(\varepsilon, \frac{\sigma}{M_{2}}\right) V^{(0)}\left(M_{1}+\phi, M_{2}+\sigma\right), \text { where } \\
& F(\varepsilon, s)=1+\varepsilon\left(a_{1} s+a_{2} s^{2}+\ldots\right)+\varepsilon^{2}\left(b_{1} s+b_{2} s^{2}+\ldots\right)+\ldots, \\
& V^{(0)}(x, y)=\frac{\lambda_{\phi}}{4 !} x^{4}+\frac{\lambda_{m}}{4} x^{2} y^{2}+\frac{\lambda_{\sigma}}{4 !} y^{4}+\sum_{n=1}^{\infty} \frac{\lambda_{(4+2 n)}}{4+2 n} \frac{x^{4+2 n}}{y^{2 n}} \equiv\left(x^{2}+y^{2}\right)^{2} W^{(0)}\left(\frac{x}{y}\right) .
\end{aligned}
$$

$F$ is assumed to be a regular function that satisfies $F(0, s)=1$ and can be Taylor-expanded in both its arguments. The finite numerical parameters $M_{1}$ and $M_{2}$ have dimension $1-\varepsilon$, and $z$ as well as $a_{i}, b_{i}, \ldots$ are dimensionless. $M_{2}$ sets the dimension of what would normally be called the renormalization scale, the quantity $z M_{2}^{\frac{1}{1-\varepsilon}}$, but the arbitrariness of its value is retained by freely choosing $z$.

Additionally, we demand that there exists a value $t_{0}$, such that $W^{(0)}\left(t_{0}\right)=0=\frac{\mathrm{d}}{\mathrm{d} t} W^{(0)}\left(t_{0}\right)$. This translates into certain conditions on the coupling constants, which we impose by hand.

In the limit $\varepsilon \rightarrow 0$ we have $F=1$ and the classical Lagrangian is seen to be scale symmetric. The above condition about existence of $t_{0}$ amounts to the assumption that there exists a flat direction in the classical potential $V^{(0)}$ and consequently a broken phase of the four-dimensional, scale symmetric model. Shifting the field variables as in $\tilde{\phi}=M_{1}+\phi, \tilde{\sigma}=M_{2}+\sigma$ makes the Lagrangian manifestly invariant under scale transformations of $\tilde{\phi}$ and $\tilde{\sigma}$, and $\phi, \sigma$ are interpreted as fluctuations around respective non-zero vacuum expectation values $\langle\tilde{\phi}\rangle=M_{1},\langle\tilde{\sigma}\rangle=M_{2}$ in the broken phase.

Yet we want to calculate loop corrections before taking the limit $\varepsilon \rightarrow 0$. This will generically unveil the anomaly of scale symmetry and the fact that the flat direction does not exist. But to proceed we need to employ yet another perturbative expansion of the (already perturbatively defined) $\mathscr{L}_{\text {eff }}$ in (2.3). Namely we restrict $\phi$ and $\sigma$ to satisfy $\phi, \sigma \ll M_{2}$, so that at each order of the calculation only a finite number of terms is relevant, up to a predefined power of $\frac{1}{M_{2}}$.

Consequently only a finite number of the $a_{i}, b_{i}, \ldots$ parameters is relevant. Different choices of these parameters (and the $F$ function in general) would result in different loop corrections. Notably, only when $F=1$ the choice $\lambda_{(4+2 n)}=0, n=1,2, \ldots$ is constant with respect to renormalization group running. Imagine that we have calculated $\mathscr{L}_{\text {eff }}$ to some order, using the background field method with

$$
\phi=\phi_{0}+\phi^{\prime}, \quad \sigma \rightarrow \sigma_{0}+\sigma^{\prime} .
$$

For example, that we have obtained $V_{\text {eff }}\left(\phi_{0}, \sigma_{0}\right)$ and $\mathscr{Z}\left(\phi_{0}, \sigma_{0}\right)$ corrected by one- and two-loop diagrams, up to $\mathscr{O}\left(\frac{\phi_{0}^{2}}{M_{2}^{2}}, \frac{\phi_{0} \sigma_{0}}{M_{2}^{2}}, \frac{\sigma_{0}^{2}}{M_{2}^{2}}\right)$. The idea behind the SI regularization is to choose the $F$ function in accordance with a requirement that $\mathscr{L}_{\text {eff }}\left(\phi_{0}, \sigma_{0}\right)$ be invariant with respect to the following transformation

$$
\begin{array}{ll}
\phi_{0} \rightarrow \phi_{0}+\Delta_{1}, & M_{1} \rightarrow M_{1}-\Delta_{1} \\
\sigma_{0} \rightarrow \sigma_{0}+\Delta_{2}, & M_{2} \rightarrow M_{2}-\Delta_{2}
\end{array}
$$

(with $z$ and $\lambda_{\phi}, \lambda_{m}, \lambda_{\sigma}, \lambda_{(4+2 n)}$ kept constant) up to the employed level of accuracy in the expansion in $\mathscr{O}\left(\frac{1}{M_{2}}\right)$, where $\Delta_{1,2}$ are are treated as constant values much smaller than $M_{2}$. While (3.5) manifestly does not change $\mathscr{L}_{\text {eff }}$, for (3.6) to be a symmetry, one has to adjust the $a_{i}, b_{i}, \ldots$ parameters. 
For example, schematically, the following combination

$$
\log \frac{\left(M_{1}+\phi\right)^{2}}{\left(z M_{2}\right)^{2}}-a_{1} \frac{\sigma}{M_{2}} \stackrel{a_{1}=2}{=} \log \frac{\left(M_{1}+\phi\right)^{2}}{\left(z\left(M_{2}+\sigma\right)\right)^{2}}+\mathscr{O}\left(\frac{\sigma^{2}}{M_{2}^{2}}\right)
$$

becomes invariant after the choice $a_{1}=2$.

When $\mathscr{L}_{\text {eff }}$ is invariant under (3.5) and (3.6), we can consistently reinterpret $M_{1}$ and $M_{2}$ as vacuum expectation values of $\phi$ and $\sigma$ (just as we did for $\mathscr{L}_{\text {class }}$ in 4 dimensions) and redefine the scale symmetry to regard these parameters as charged, so that the Lagrangian is now symmetric with respect to

$$
\begin{aligned}
& M_{1}+\phi(x) \rightarrow s^{1-\varepsilon}\left(M_{1}+\phi(s x)\right) \\
& M_{2}+\sigma(x) \rightarrow s^{1-\varepsilon}\left(M_{2}+\sigma(s x)\right)
\end{aligned}
$$

The straightforward observation at this point is that this will automatically be true to all orders, if we choose

$$
F(\varepsilon, s)=(1+s)^{\frac{2 \varepsilon}{1-\varepsilon}}
$$

so that

$$
\mathscr{L}_{\text {class }}=\frac{1}{2}(\partial \phi)^{2}+\frac{1}{2}(\partial \sigma)^{2}-\left(z\left(M_{2}+\sigma\right)^{\frac{1}{1-\varepsilon}}\right)^{2 \varepsilon} V^{(0)}\left(M_{1}+\phi, M_{2}+\sigma\right) .
$$

Due to the perturbative expansion in $1 / M_{2}$, (3.11) is no more consequential than fixing a finite number of $a_{i}, b_{i}, \ldots$ parameters by hand. The qualitative novelty achieved this way is the possibility to consistently assume that the model describes a broken phase of a scale symmetric, anomaly-free theory. That alone is a nonperturbative characteristic, as it for example predicts the presence of a massless Goldstone mode.

\section{Counterterms and renormalization group}

To regularize $\mathscr{L}_{\text {eff }}$ calculated from (3.11), one introduces bare fields and couplings. The counterterms needed are: one for each field plus one for each $\lambda$ coupling in $V^{(0)}$. Additionally, there is no point in keeping non-zero values of $M_{1}$ and $M_{2}$ on top of using the background field method. In other words, loop corrections can be computed in the unbroken phase. Thus, we have

$$
\begin{aligned}
& \tilde{\phi}=\phi=\phi_{0}+\phi^{\prime}, \quad \tilde{\sigma}=\sigma=\sigma_{0}+\sigma^{\prime}, \\
& \phi^{B}=Z_{\phi} \phi, \quad \sigma^{B}=Z_{\sigma} \sigma, \quad \lambda_{N}^{B}=Z_{\lambda_{N}} \lambda_{N}, \quad N=\phi, m, \sigma, 6,8, \ldots \\
& \mathscr{L}=\frac{1}{2} Z_{\phi}(\partial \phi)^{2}+\frac{1}{2} Z_{\sigma}(\partial \sigma)^{2} \\
& -\mu(\sigma)^{2 \varepsilon}\left(Z_{\lambda_{\phi}} \frac{\lambda_{\phi}}{4 !} \phi^{4}+Z_{\lambda_{m}} \frac{\lambda_{m}}{4} \phi^{2} \sigma^{2}+Z_{\lambda_{\sigma}} \frac{\lambda_{\sigma}}{4 !} \sigma^{4}+\sum_{n=1}^{\infty} Z_{\lambda_{(4+2 n)}} \frac{\lambda_{(4+2 n)}}{4+2 n} \frac{\phi^{4+2 n}}{\sigma^{2 n}}\right) \\
& \mu(\sigma)=z \sigma^{\frac{1}{1-\varepsilon}}
\end{aligned}
$$

The choice where Z's contain no finite terms beyond the zeroth order,

$$
Z=1+\frac{\delta}{\varepsilon}+\ldots
$$


defines the minimal subtraction scheme.

Notably, even upon choosing $\lambda_{(4+2 n)}=0, n=1,2, \ldots$, the usual five counterterms, $Z_{\phi}, Z_{\sigma}, Z_{\lambda_{\phi}}$, $Z_{\lambda_{m}}$ and $Z_{\lambda_{\sigma}}$, are not enough to regularize the model. Divergencies of the type $\frac{1}{\varepsilon} \frac{\phi_{0}^{4+2 n}}{\sigma_{0}^{2 n}}$ will inevitably be produced in loop diagrams with the evanescent interactions stemming from the expansion of $\sigma^{\frac{2 \varepsilon}{1-\varepsilon}}$.

In the previuos section we had discussed the choice where $\mu=\mu(\tilde{\sigma})$ is a function of the $\tilde{\sigma}$ field alone. But it also clear that any dimension 1 function of both fields, $\mu(\tilde{\phi}, \tilde{\sigma})$, would be equally good for ensuring the invariance with respect to (3.8) and (3.9). But such a general choice would result in the need to introduce interaction terms (and corresponding counterterms) with both $\sigma$ and $\phi$ in a denominator. Consequently the perturbative expansion of $\mathscr{L}_{\text {eff }}$ would be valid in a still smaller fraction of the field space where $\phi, \sigma \ll M_{1}, M_{2}$. There, presumably, physical results do not depend on what $\mu$ function was chosen. The ability to freely choose this function should be part of the general freedom of choosing a regularization method. Sticking to our choice in (4.5), this freedom is reduced to arbitrariness of the $z$ parameter.

The requirement that the bare couplings, $\lambda^{B}$ 's do not depend on $z$ leads in a familiar way to the running of renormalized couplings, $\lambda=\lambda(z)$,

$$
\lambda_{B}=z^{2 \varepsilon} Z_{\lambda} \lambda(z) Z_{\phi}^{-n} Z_{\sigma}^{-\left(m+\frac{\varepsilon}{1-\varepsilon}\right)}
$$

where $n$ and $m$ are numbers appropriate for a given $\lambda$, for example $n=3, m=-1$ for $\lambda_{6}$. Let us specify to the case of minimal subtraction below. The superscript in parenthesis denotes order in $\lambda$ 's.

$$
\begin{aligned}
& \frac{\mathrm{d} \lambda}{\mathrm{d} \log z}=-2 \varepsilon \lambda+\beta_{\lambda}(\{\lambda\}) \\
& 0=\frac{\mathrm{d} \log \lambda_{B}}{\mathrm{~d} \log z}=2 \varepsilon+\frac{\mathrm{d} \log Z_{\lambda}}{\mathrm{d} \log z}+\frac{1}{\lambda(z)} \frac{\mathrm{d} \lambda}{\mathrm{d} \log z}-n \frac{\mathrm{d} \log Z_{\phi}}{\mathrm{d} \log z}-\left(m+\frac{\varepsilon}{1-\varepsilon}\right) \frac{\mathrm{d} \log Z_{\phi}}{\mathrm{d} \log z} \\
& \Rightarrow \frac{\mathrm{d} \lambda}{\mathrm{d} \log z}=-2 \varepsilon \lambda-\lambda \sum_{i=\phi, m, \sigma, 6, \ldots}\left[\frac{1}{\varepsilon}\left(\frac{\partial \delta_{\lambda}^{(1)}}{\partial \lambda_{i}}-n \frac{\partial \delta_{\phi}^{(1)}}{\partial \lambda_{i}}-\left(m+\frac{\varepsilon}{1-\varepsilon}\right) \frac{\partial \delta_{\sigma}^{(1)}}{\partial \lambda_{i}}\right)+\mathscr{O}(\lambda)\right] \frac{\mathrm{d} \lambda_{i}}{\mathrm{~d} \log z}
\end{aligned}
$$

Equation (4.10) is solved for $\frac{\mathrm{d} \lambda_{i}}{\mathrm{~d} \log z}$ perturbatively in an iterative manner. Here we stopped the expansion in powers of lambdas at the lowest order, but there is of course no reason to do that, assuming one has calculated higher-order counterterms, $\delta^{(2)}$ etc. The result is

$$
\begin{aligned}
\beta_{\lambda} & =2 \lambda \sum_{i} \lambda_{i}\left(\frac{\partial \delta_{\lambda}^{(1)}}{\partial \lambda_{i}}-n \frac{\partial \delta_{\phi}^{(1)}}{\partial \lambda_{i}}-m \frac{\partial \delta_{\sigma}^{(1)}}{\partial \lambda_{i}}\right) \\
& +2 \lambda \sum_{i} \lambda_{i} \frac{\partial \delta_{\sigma}^{(1)}}{\partial \lambda_{i}}\left[\sum_{j} \lambda_{j}\left(\frac{\partial \delta_{\lambda_{i}}^{(1)}}{\partial \lambda_{j}}-n_{i} \frac{\partial \delta_{\phi}^{(1)}}{\partial \lambda_{i}}-m_{i} \frac{\partial \delta_{\sigma}^{(1)}}{\partial \lambda_{i}}\right)\right]+\mathscr{O}\left(\lambda^{3}\right),
\end{aligned}
$$

where the last explicit contribution is already of order $\mathscr{O}\left(\lambda^{3}\right)$ and should have been omitted at the lowest order. We display it, because it is a contribution that originates from the fact that $Z_{\sigma}$ is raised 
to power $m+\frac{\varepsilon}{1-\varepsilon}$ rather than $m$ in (4.7) ${ }^{1}$. It is new relative to the case of standard dimensional regularization with $\mu=$ const.

Thus, there are two ways in which the fact that we are using the SI regularization manifests itself in the renormalization group functions. Firstly, the evanescent power of the field $\sigma$ in each interaction results in a contribution from this field's anomalous dimension to every coupling, as we see in (4.11). Secondly, starting from the level of two-loops, there will be new counterterms originating from the evanescent interactions proportional to the non-zero $a_{i}, b_{i}, \ldots$ in (3.2). Among those new counterterms, there will be poles of the form $\frac{1}{\varepsilon} \frac{\phi^{4+2 n}}{\sigma^{2 n}}$. Hence, even if the couplings $\lambda_{(4+2 n)}$ are all set to zero at some $z=z_{0}$, nonpolynomial interactions will be generated at a different $z$.

The issue of an apparent contradiction in the presence of both, running couplings and nonanomalous scale symmetry was discussed in [15]. The SI regularization method does not spoil validity of the Callan-Symanzik equation. In comparison with the $\overline{M S}$ prescription with the standard dimensional regularization, one has new contributions both to $\mathscr{L}_{\text {eff }}$ and to the renormalization group functions, but the modifications work together so that one still has

$$
\frac{\mathrm{d}}{\mathrm{d} z} \mathscr{L}_{\text {eff }}=0
$$

This was explicitly shown in [16] for the two-loop effective potential in the model of two scalars that we have considered here.

\section{Conclusions}

We have discussed a scale symmetry respecting method of dimensionally regularizing ultraviolet divergencies in a quantum field theory. The method can be succinctly characterized as promoting the renormalization scale $\mu$ to a dynamical quantum field. But we propose to reinterpret it as a way of organizing one's loop calculation in a model with nonrenormalizable interactions. The latter can be introduced is such a way that their suppression scale as well as $\mu$ can consistently be defined as vacuum expectation value of a dynamical scalar field present in the Lagrangian. This way, at the cost of nonrenormalizability, a model can be interpreted as a description of interactions in the broken phase of scale symmetry. We have discussed the notion of running couplings in this setup and pointed out generic modifications in renormalization group equations relative to the case of calculating loop corrections to the same classical Lagrangian using the usual dimensional regularization with $\mu=$ const.

\section{Ackowledgemends}

This work has been supported by National Science Centre, Poland, under research grant DEC2016/21/N/ST2/03312.

\footnotetext{
${ }^{1}$ In the case of our Lagrangian (4.4) where there are only scalar fields, $\delta_{\phi}^{(1)}$ and $\delta_{\sigma}^{(1)}$ happen to be zero as there is no field renormalization at one-loop. This would no longer be true if for example $\sigma$ were coupled to a fermion. In any case the discussion is relevant for higher orders as well, hence we keep $\delta_{\phi, \sigma}^{(1)}$ for simplicity of the presentation.
} 


\section{References}

[1] J. Iliopoulos, C. Itzykson and A. Martin, Functional Methods and Perturbation Theory, Rev. Mod. Phys. 47 (1975) 165. doi:10.1103/RevModPhys.47.165

[2] Chapter 33. in M. D. Schwartz, Quantum Field Theory and the Standard Model, Cambridge University Press, ISBN: 9781107034730

[3] M. E. Shaposhnikov and I. I. Tkachev, Quantum scale invariance on the lattice, Phys. Lett. B 675 (2009) 403 doi:10.1016/j.physletb.2009.04.040 [arXiv:0811.1967 [hep-th]].

[4] C. M. Fraser, Calculation of Higher Derivative Terms in the One Loop Effective Lagrangian, Z. Phys. C 28 (1985) 101. doi:10.1007/BF01550255

[5] Appendix D in A. Andreassen, D. Farhi, W. Frost and M. D. Schwartz, Precision decay rate calculations in quantum field theory, Phys. Rev. D 95 (2017) no.8, 085011 doi:10.1103/PhysRevD.95.085011 [arXiv:1604.06090 [hep-th]].

[6] F. Englert, C. Truffin and R. Gastmans, Conformal Invariance in Quantum Gravity, Nucl. Phys. B 117 (1976) 407. doi:10.1016/0550-3213(76)90406-5

[7] E. S. Fradkin and G. A. Vilkovisky, Conformal Off Mass Shell Extension and Elimination of Conformal Anomalies in Quantum Gravity, Phys. Lett. 73B (1978) 209. doi:10.1016/0370-2693(78)90838-9

[8] M. Shaposhnikov and D. Zenhausern, Quantum scale invariance, cosmological constant and hierarchy problem, Phys. Lett. B 671 (2009) 162 doi:10.1016/j.physletb.2008.11.041 [arXiv:0809.3406 [hep-th]].

[9] R. Armillis, A. Monin and M. Shaposhnikov, Spontaneously Broken Conformal Symmetry: Dealing with the Trace Anomaly, JHEP 1310 (2013) 030 doi:10.1007/JHEP10(2013)030 [arXiv:1302.5619 [hep-th]].

[10] F. Gretsch and A. Monin, Perturbative conformal symmetry and dilaton, Phys. Rev. D 92 (2015) no.4, 045036 doi:10.1103/PhysRevD.92.045036 [arXiv:1308.3863 [hep-th]].

[11] K. Allison, C. T. Hill and G. G. Ross, Ultra-weak sector, Higgs boson mass, and the dilaton, Phys. Lett. B 738 (2014) 191 doi:10.1016/j.physletb.2014.09.041 [arXiv:1404.6268 [hep-ph]].

[12] M. E. Shaposhnikov and F. V. Tkachov, Quantum scale-invariant models as effective field theories, arXiv:0905.4857 [hep-th].

[13] D. M. Ghilencea, Manifestly scale-invariant regularization and quantum effective operators, Phys. Rev. D 93 (2016) no.10, 105006 doi:10.1103/PhysRevD.93.105006 [arXiv:1508.00595 [hep-ph]].

[14] D. M. Ghilencea, Z. Lalak and P. Olszewski, Standard Model with spontaneously broken quantum scale invariance, arXiv:1612.09120 [hep-ph].

[15] C. Tamarit, Running couplings with a vanishing scale anomaly, JHEP 1312 (2013) 098 doi:10.1007/JHEP12(2013)098 [arXiv:1309.0913 [hep-th]].

[16] D. M. Ghilencea, Z. Lalak and P. Olszewski, Two-loop scale-invariant scalar potential and quantum effective operators, Eur. Phys. J. C 76 (2016) no.12, 656 doi:10.1140/epjc/s10052-016-4475-0 [arXiv:1608.05336 [hep-th]]. 